\title{
The combinatorics of interval-vector polytopes
}

\author{
Matthias Beck \\ Department of Mathematics \\ San Francisco State University \\ San Francisco, U.S.A. \\ mattbeck@sfsu.edu \\ Gabriel Dorfsman-Hopkins \\ Department of Mathematics \\ Dartmouth College \\ Hanover, U.S.A.
}

\author{
Jessica De Silva \\ Department of Mathematics \\ University of Nebraska \\ Lincoln, U.S.A.
}

Gabriel.D.Dorfsman-Hopkins.13@dartmouth.edu

jessica.desilva@huskers.unl.edu

Joseph Pruitt

Department of Mathematics

University of Illinois

Urbana-Champaign, U.S.A.

j92pruitt@gmail.com

Submitted: Dec 18, 2012; Accepted: Aug 12, 2013; Published: Aug 23, 2013

Mathematics Subject Classifications: 52B05, 05A15, 52B20

\begin{abstract}
An interval vector is a $(0,1)$-vector in $\mathbb{R}^{n}$ for which all the 1 's appear consecutively, and an interval-vector polytope is the convex hull of a set of interval vectors in $\mathbb{R}^{n}$. We study three particular classes of interval vector polytopes which exhibit interesting geometric-combinatorial structures; e.g., one class has volumes equal to the Catalan numbers, whereas another class has face numbers given by the Pascal 3-triangle.
\end{abstract}

Keywords: Interval vector, lattice polytope, Ehrhart polynomial, root polytope, Catalan number, $f$-vector 


\section{Introduction}

An interval vector is a $(0,1)$-vector $x \in \mathbb{R}^{n}$ such that, if $x_{i}=x_{k}=1$ for $i<k$, then $x_{j}=1$ for every $i \leqslant j \leqslant k$. In [2] Dahl introduced the class of interval-vector polytopes, which are formed by taking the convex hull of a set of interval vectors in $\mathbb{R}^{n}$. Our goal is to derive combinatorial properties of certain interval-vector polytopes.

For $i \leqslant j$, let $\alpha_{i, j}:=e_{i}+e_{i+1}+\cdots+e_{j}$, where $e_{i}$ is the $i^{t h}$ standard unit vector. The interval length of $\alpha_{i j}$ is $j-i+1$. Let $S \subset \mathbb{N}$. For a fixed $n$, let $\mathcal{I}_{S}$ be the set of interval vectors in $\mathbb{R}^{n}$ with interval length in $S$. (If $S$ is small, we may leave out the brackets in the set notation; e.g., we will denote $\mathcal{I}_{\{i, j\}}$ by $\mathcal{I}_{i, j}$.) We will denote the set of all non-zero interval vectors in a given dimension as $\mathcal{I}_{[n]}$. Let $\mathcal{P}_{n}\left(\mathcal{I}_{S}\right)$ be the convex hull of $\mathcal{I}_{S} \subset \mathbb{R}^{n}$.

There are three classes of interval vector polytopes that we will consider in this paper. In Section 3 we study the complete interval vector polytope $\mathcal{P}_{n}\left(\mathcal{I}_{[n]}\right)$, the convex hull of all interval vectors in $\mathbb{R}^{n}$ except the zero vector. In Section 4 we look at the fixed interval vector polytope $\mathcal{P}_{n}\left(\mathcal{I}_{i}\right)$ given by the convex hull of all interval vectors with interval length $i$. In Section 5 we introduce the first in a class of pyramidal interval polytopes: the first pyramidal interval vector polytope $\mathcal{P}_{n}\left(\mathcal{I}_{1, n-1}\right)$, the convex hull of all interval vectors in $\mathbb{R}^{n}$ with interval length 1 or $n-1$. (The reason for the term pyramidal interval polytope will also become clear in Section 5.) In Section 6 we generalize this to the $i^{\text {th }}$ pyramidal interval vector polytope $\mathcal{P}_{n}\left(\mathcal{I}_{1, n-i}\right)$. We examine combinatorial characteristics of these polytopes such as the $f$-vector and volume and discover unexpected relations to wellknown numerical sequences.

Let $t$ be a positive integer variable. For a lattice polytope $\mathcal{P}$ (i.e., the vertices of $\mathcal{P}$ all have integer coordinates), the Ehrhart polynomial $L_{\mathcal{P}}(t)$ is the counting function yielding the number of lattice points in $t \mathcal{P}:=\{t v \mid v \in \mathcal{P}\}$. Ehrhart [5] proved that $L_{\mathcal{P}}(t)$ is indeed a polynomial; see, e.g., [1] for more about Ehrhart polynomials. The Ehrhart polynomial contains useful geometric information about a polytope; in particular, the leading coefficient of the Ehrhart polynomial gives the volume of the polytope.

In [9], Postnikov defines the complete root polytope $Q_{n} \subset \mathbb{R}^{n}$ as the convex hull of

0 and $e_{i}-e_{j}$ for all $i<j$ where $e_{i}$ is the $i^{\text {th }}$ standard unit vector. He showed (among many other things) that the volume of $Q_{n+1}$ is $C_{n}:=\frac{1}{n+1}\left(\begin{array}{c}2 n \\ n\end{array}\right)$, the $n^{\text {th }}$ Catalan number. In Section 3 we prove, in a discrete-geometric sense, that $Q_{n+1}$ and the complete interval vector polytope $\mathcal{P}_{n}\left(\mathcal{I}_{[n]}\right)$ are interchangeable, that is, the two polytopes have the same Ehrhart polynomial.

Theorem 1. $L_{Q_{n+1}}(t)=L_{\mathcal{P}_{n}\left(\mathcal{I}_{[n]}\right)}(t)$.

Corollary 2. The volume of the complete interval vector polytope $\mathcal{P}_{n}\left(\mathcal{I}_{[n]}\right)$ equals the $n^{\text {th }}$ Catalan number.

A unimodular simplex in $\mathbb{R}^{d}$ is an $n$-dimensional lattice simplex $\Delta$ whose edge direction at any vertex form a lattice basis for $\mathbb{Z}^{d} \cap \operatorname{aff}(\Delta)$, where aff $(\Delta)$ is the affine hull of $\Delta$. In Section 4 we prove:

Theorem 3. The fixed interval vector polytope $\mathcal{P}_{n}\left(\mathcal{I}_{i}\right)$ is an $(n-i)$-dimensional unimodular simplex. 
Given an $n$-dimensional polytope $\mathcal{P}$ with $f_{k} k$-dimensional faces, the $f$-vector of $\mathcal{P}$ is written as $f(\mathcal{P}):=\left(f_{-1}, f_{0}, f_{1}, \ldots, f_{n}\right)$ where $f_{-1}, f_{n}:=1$ (see, e.g., [7] for more about $f$-vectors). In Section 5 we show:

Theorem 4. For $n \geqslant 3$, the $f$-vector of the first pyramidal interval vector polytope satisfies $f_{k}\left(\mathcal{P}_{n}\left(\mathcal{I}_{1, n-1}\right)\right)=\left(\begin{array}{c}n-1 \\ k\end{array}\right)+\left(\begin{array}{c}n+1 \\ k+1\end{array}\right)$.

The $f$-vector of $\mathcal{P}_{n}\left(\mathcal{I}_{1, n-1}\right)$ is thus the $n^{\text {th }}$ row of the Pascal 3-triangle (see, e.g., $[10$, Sequence A028262]), in particular, it is symmetric. We also show that the volume of the $1^{\text {st }}$ pyramidal interval vector polytope is simple:

Theorem 5. For $n \geqslant 3, \operatorname{vol}\left(\mathcal{P}_{n}\left(\mathcal{I}_{1, n-1}\right)\right)=2(n-2)$.

Finally, in Section 6 we lay out future work on $i^{\text {th }}$ pyramidal interval vector polytopes.

\section{Preliminaries}

In this paper, we will be analyzing the properties of certain classes of convex polytopes which are formed by taking the convex hull of finitely many points in $\mathbb{R}^{n}$. The convex hull of a set $A=\left\{v_{1}, v_{2}, \ldots, v_{m}\right\} \subset \mathbb{R}^{n}$, denoted $\operatorname{conv}(A)$, is defined as

$$
\left\{\lambda_{1} v_{1}+\lambda_{2} v_{2}+\cdots+\lambda_{m} v_{m} \mid \lambda_{1}, \lambda_{2}, \ldots, \lambda_{m} \in \mathbb{R}_{\geqslant 0} \text { and } \sum_{i=1}^{m} \lambda_{i}=1\right\} .
$$

The polytope $\operatorname{conv}(A)$ is contained in the affine hull aff $(A)$ of $A$, defined as in (1) but without the restriction that $\lambda_{1}, \lambda_{2}, \ldots, \lambda_{m} \geqslant 0$. We call a set of points affinely (resp. convexly) independent if each point is not in the affine (resp. convex) hull of the rest. The vertex set of a polytope is the minimal convexly independent set of points whose convex hull form the polytope. A polytope is d-dimensional if the dimension of its affine hull is $d$. We denote the dimension of the polytope $\mathcal{P}$ as $\operatorname{dim}(\mathcal{P})$. We call a $d$-dimensional polytope a $d$-simplex if it has $d+1$ vertices.

A lattice point is a point with integral coordinates. A lattice polytope is a polytope whose vertices are lattice points. The normalized volume of a polytope $\mathcal{P}$, denoted $\operatorname{vol}(\mathcal{P})$, is the volume with respect to a unimodular simplex (recall definition in Section 1). We will refer to the normalized volume of a polytope as its volume. Note that the leading coefficient of the Ehrhart polynomial of a lattice polytope $\mathcal{P}$ is $\frac{1}{d !} \operatorname{vol}(\mathcal{P})$.

A hyperplane is a set of the form

$$
H:=\left\{x \in \mathbb{R}^{n} \mid a_{1} x_{1}+\cdots+a_{n} x_{n}=b\right\},
$$

where not all $a_{j}$ 's are 0 . The half-spaces defined by this hyperplane are formed by the two weak inequalities corresponding to the equation defining the hyperplane. A face of $\mathcal{P}$ is the intersection of a hyperplane and $\mathcal{P}$ such that $\mathcal{P}$ lies completely in one halfspace of the hyperplane. This face is a polytope called a $k$-face if its dimension is $k$. A vertex is a 0 -face and an edge is a 1 -face. Given an $n$-dimensional polytope $\mathcal{P}$ with 
$f_{k} k$-dimensional faces, the $f$-vector of $\mathcal{P}$ is written as $f(\mathcal{P}):=\left(f_{-1}, f_{0}, \ldots, f_{n}\right)$. For example, a triangle $\triangle$ is a 2-dimensional polytope with 3 vertices and 3 edges and thus has $f$-vector $f(\triangle)=(1,3,3,1)$.

\section{Complete Interval Vector Polytopes}

In [2] Dahl provides a method for determining the dimension of these polytopes which we will use throughout this paper. We utilized the software packages polymake [6] and LattE $[4,8]$ to find most of the patterns described by our results.

Proof of Theorem 1. Each of the vertices of $Q_{n}$ are vectors with entries that sum to zero, so any linear combination (and specifically any convex combination) of these vertices also has entries who sum to zero. Define $B:=\left\{x \in \mathbb{R}^{n} \mid \sum_{i=1}^{n} x_{i}=0\right\}$; thus $Q_{n} \subset B$, and $B$ is an $(n-1)$-dimensional affine subspace of $\mathbb{R}^{n}$.

Consider the linear transformation $T$ given by the $n \times n$ lower triangular matrix with entries $t_{i, j}=1$ if $i \geqslant j$ and $t_{i, j}=0$ otherwise. Then

$$
T(B) \subseteq A:=\left\{x \in \mathbb{R}^{n} \mid x_{n}=0\right\} .
$$

Since (the matrix representing) $T$ has determinant 1 , it is injective when restricting the domain to $B$. For the same reason, we know that for any $y \in A$, there exists $x \in \mathbb{R}^{n}$ such that $y=T(x)$. But since $y_{n}=\sum_{i=1}^{n} x_{i}=0$, then $x \in B$, so that $\left.T\right|_{B}: B \rightarrow A$ is surjective, and therefore a linear bijection.

Also, the projection $\Pi: A \rightarrow \mathbb{R}^{n-1}$ given by

$$
\Pi\left(\left(x_{1}, \ldots, x_{n-1}, 0\right)\right)=\left(x_{1}, \ldots, x_{n-1}\right),
$$

is clearly a linear bijection.

Now we show that the linear bijection $\left.\Pi \circ T\right|_{B}: B \rightarrow \mathbb{R}^{n-1}$ is a lattice-preserving map, i.e., an isomorphism from $B \cap \mathbb{Z}^{n}$ to $\mathbb{Z}^{n-1}$ (viewed as additive groups). First we find a lattice basis for $B$. Consider

$$
C:=\left\{e_{i, n}=e_{i}-e_{n} \mid i<n\right\}
$$

We notice that any integer point of $B$ can be represented as

$$
\left(a_{1}, \ldots, a_{n-1},-\sum_{i=1}^{n-1} a_{i}\right)=\sum_{i=1}^{n-1} a_{i} e_{i, n}
$$

and so $C$ is a lattice basis.

Note that $\Pi \circ T\left(e_{i, n}\right)=e_{i}+\cdots+e_{n-1}=: u_{i}$. Therefore

$$
\Pi \circ T(C)=\left\{u_{i} \mid i \leqslant n-1\right\}=: U \text {. }
$$

We notice that $e_{n-1}=u_{n-1}$ and $e_{i}=u_{i}-u_{i+1}$, so that each of the standard unit vectors $e_{i}$ of $\mathbb{R}^{n-1}$ is an integral combination of the vectors in $U$. Since the standard basis is a 
lattice basis, so is $U$, thus $\left.\Pi \circ T\right|_{B}$ is a lattice-preserving map. Since our bijection is linear and lattice-preserving, all we have left to show is that the vertices of $Q_{n}$ map to those of $\mathcal{P}_{n-1}\left(\mathcal{I}_{[n-1]}\right)$. By linearity, $\Pi \circ T(0)=0$, and given any vertex $\alpha_{i, j}$ of $\mathcal{P}_{n-1}\left(\mathcal{I}_{[n-1]}\right)$, we know that $\Pi \circ T\left(e_{i, j+1}\right)=\alpha_{i, j}$ where $i<j+1 \leqslant n$ so that $\left.\Pi \circ T\right|_{B}$ maps vertices to vertices.

Corollary 2 follows directly from this theorem and [9], since the leading coefficient of the Ehrhart polynomial of $\mathcal{P}_{n}$ is $\frac{1}{n !}$ times the volume of $\mathcal{P}_{n}$.

\section{Fixed Interval Vector Polytopes}

The following construction is due to [2]. We define the set of elementary vectors as containing all $e_{i, j}=e_{i}-e_{j}$, each unit vector $e_{i}$, and the zero vector. Let $T$ be the lower triangular matrix from the proof of Theorem 1. We notice that $T\left(e_{i}\right)=\alpha_{i, n}$ and $T\left(e_{i, j}\right)=\alpha_{i, j-1}$. So the image of an elementary vector is an interval vector. Since $T$ is invertible, for any set of interval vectors $\mathcal{I}$, there is a unique set $\mathcal{E}$ of elementary vectors such that $T(\mathcal{E})=\mathcal{I}$, namely $\mathcal{E}=T^{-1}(\mathcal{I})$.

Thus for any interval vector polytope $\mathcal{P}_{n}\left(\mathcal{I}_{S}\right) \subset \mathbb{R}^{n}$, we can construct the corresponding flow-dimension graph $G_{\mathcal{I}_{S}}=(V, E)$ as follows. Let $\mathcal{E}_{S}=T^{-1}\left(\mathcal{I}_{S}\right)$. Let the vertex set $V=[n]$. Specify a subset $V_{1}=\left\{j \in V \mid e_{j} \in \mathcal{E}_{S}\right\}$, and define the directed edge set $E=\left\{(i, j) \mid e_{i, j} \in \mathcal{E}_{S}\right\}$. Let $k_{0}$ denote the number of connected components $\mathcal{C}$ of the graph $G$ (ignoring direction) so that $\mathcal{C} \cap V_{1}$ is empty.

Recall that the fixed interval vector polytope $\mathcal{P}_{n}\left(\mathcal{I}_{i}\right)$ is the convex hull of all interval vectors in $\mathbb{R}^{n}$ with interval length $i$. For example, the fixed interval vector polytope with $n=5, i=3$ is

$$
\mathcal{P}_{5}\left(\mathcal{I}_{3}\right)=\operatorname{conv}((1,1,1,0,0),(0,1,1,1,0),(0,0,1,1,1))
$$

and its flow-dimension graph is depicted in Figure 1.

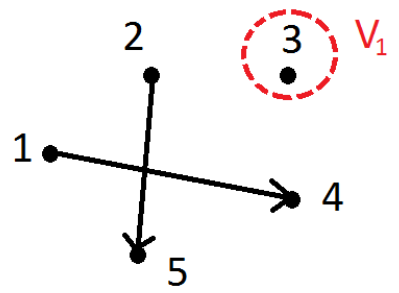

Figure 1: The flow-dimension graph of $\mathcal{P}_{5}\left(\mathcal{I}_{3}\right)$.

Theorem 6 (Dahl [2]). If $0 \in \operatorname{aff}\left(\mathcal{I}_{S}\right)$, then the dimension of $\mathcal{P}_{n}\left(\mathcal{I}_{S}\right)$ is $n-k_{0}$. Else, if $0 \notin \operatorname{aff}\left(\mathcal{I}_{S}\right)$ then the dimension of $\mathcal{P}_{n}\left(\mathcal{I}_{S}\right)$ is $n-k_{0}-1$. 
For a fixed $i$,

$$
T^{-1}\left(\mathcal{I}_{i}\right)=\mathcal{E}_{i}=\left\{e_{k, k+i} \mid k \leqslant n-i\right\} \cup\left\{e_{n-i+1}\right\} .
$$

The corresponding flow-dimension graph is $G_{\mathcal{P}_{n}\left(\mathcal{I}_{i}\right)}=(V, E)$ where $V=\{1, \ldots, n\}$ and $E=\{(k, k+i) \mid k \in[n-i]\}$. Then $V_{1}=\{n-i+1\}$ corresponds to $e_{n-i+1} \in \mathcal{E}_{i}$.

Two nodes $a, b$ in a graph $G=(V, E)$ are said to be connected if there exists a path from $a$ to $b$, that is there exist $q_{0}, \ldots, q_{s} \in V$ such that $\left(a, q_{0}\right),\left(q_{0}, q_{1}\right), \ldots,\left(q_{s}, b\right) \in E$.

Lemma 7. Let $a, b$ be nodes in the flow-dimension graph $G_{\mathcal{P}_{n}\left(\mathcal{I}_{i}\right)}$. Then $a$ and $b$ are connected if and only if $a \equiv b \bmod i$.

Proof. The edges in $G_{\mathcal{P}_{n}\left(\mathcal{I}_{i}\right)}$ are of the form $(k, k+i)$, and therefore the nodes of a path in $G_{\mathcal{P}_{n}\left(\mathcal{I}_{i}\right)}$ are all in the same congruence class modulo $i$.

Proposition 8. $\mathcal{P}_{n}\left(\mathcal{I}_{i}\right)$ is an $(n-i)$-dimensional simplex.

Proof. For a given dimension and interval length, an interval vector is uniquely determined by the location of the first 1 , hence we can determine the number of vertices of $\mathcal{P}_{n}\left(\mathcal{I}_{i}\right)$ by counting all possible placements of the first 1 in an interval of $i 1$ 's. Since the string must have length $i$, the number of spaces before the first 1 must not exceed $n-i$ and so there are $n-i+1$ possible locations for the first 1 in the interval to be placed. Thus, $\mathcal{P}_{n}\left(\mathcal{I}_{i}\right)$ has $n-i+1$ vertices.

By Lemma 7 we know there are $i$ connected components in the flow-dimension graph $G_{\mathcal{P}_{n}\left(\mathcal{I}_{i}\right)}$ and since $V_{1}$ has only one element, $k_{0}=i-1$. Thus by Theorem 6 the dimension of $\mathcal{P}_{n}\left(\mathcal{I}_{i}\right)$ is $n-i$. Therefore $\mathcal{P}_{n}\left(\mathcal{I}_{i}\right)$ is an $(n-i)$-dimensional simplex.

Proof of Theorem 3. It remains to show that $\mathcal{P}_{n}\left(\mathcal{I}_{i}\right)$ is unimodular. Consider the affine space where the sum over every $i^{\text {th }}$ coordinate is 1 ,

$$
A=\left\{\mathrm{x} \in \mathbb{R}^{n} \mid \sum_{j \equiv k \bmod i} x_{j}=1, \text { for all } k \in[i]\right\} .
$$

Since the vertices of $\mathcal{P}_{n}\left(\mathcal{I}_{i}\right)$ have interval length $i$, they are in $A$; thus $\mathcal{P}_{n}\left(\mathcal{I}_{i}\right) \subset A$. We want to show that the following vectors in $\mathcal{P}_{n}\left(\mathcal{I}_{i}\right)$ form a lattice basis for $A$ :

$$
\begin{aligned}
w_{1}= & \alpha_{1, i}-\alpha_{n-i+1, n} \\
w_{2}= & \alpha_{2, i+1}-\alpha_{n-i+1, n} \\
& \vdots \\
w_{n-i}= & \alpha_{n-i, n-1}-\alpha_{n-i+1, n} .
\end{aligned}
$$

We will do this by showing that any integer point $p \in A$ can be expressed as an integral linear combination of the proposed lattice basis, that is, there exist integer coefficients $Y_{1}, \ldots, Y_{n-i}$ so that $p=Y_{1} w_{1}+\cdots+Y_{n-i} w_{n-i}+\alpha_{n-i+1, n}$.

We first notice that $p$ can be expressed as

$$
\left(p_{1}, p_{2}, \ldots, p_{n-i}, \sum_{\substack{j \leqslant n-i \\ j \equiv t-i+1 \bmod i}}\left(-p_{j}\right)+1, \sum_{\substack{j \leqslant n-i \\ j \equiv t-i+2 \bmod i}}\left(-p_{j}\right)+1, \ldots, \sum_{\substack{j \leqslant n-i \\ j \equiv t \bmod i}}\left(-p_{j}\right)+1\right)
$$


by solving for the last term in each of the equations defining $A$. Let

$$
Y_{t}= \begin{cases}p_{1} & \text { if } t=1 \\ p_{t}-p_{t-1} & \text { if } \quad 1<t \leqslant i \\ p_{t}-Y_{t-i} & \text { if } \quad i<t \leqslant n-i\end{cases}
$$

Then each $Y_{t}$ is an integer. We claim that

$$
Y_{1} w_{1}+\cdots+Y_{n-i} w_{n-i}+\alpha_{n-i+1, n}=p .
$$

Clearly the first coordinate is $p_{1}$ since $w_{1}$ is the only vector with an element in the first coordinate. Next consider the $t^{\text {th }}$ coordinate of this linear combination for $1<t \leqslant i$, by summing the coefficients of all the vectors who have a 1 in the $t^{\text {th }}$ position:

$$
Y_{t}+Y_{t-1}+Y_{t-2}+\cdots+Y_{1}=p_{t}-p_{t-1}+p_{t-1}-p_{t-2}+\cdots+p_{2}-p_{1}+p_{1}=p_{t}
$$

We next consider the $t^{\text {th }}$ coordinate of the combination for $i<t \leqslant n-i$ by summing the coefficients of the vectors who have a 1 in the $t^{\text {th }}$ position.

$$
Y_{t}+Y_{t-1}+\cdots+Y_{t-i+1}=\left(p_{t}-Y_{t-1}-\cdots-Y_{t-i+1}\right)+Y_{t-1}+\cdots+Y_{t-i+1}=p_{t}
$$

Finally, we consider the $t^{\text {th }}$ coordinate of the combination for $n-i<t \leqslant n$, noticing that each coordinate from $w_{1}$ to $w_{t}$ has a -1 in the $(t-i)^{\text {th }}$ position, and $\alpha_{n-i+1, n}$ has a 1 in this position. This gives

$$
-\left(Y_{1}+Y_{2}+\cdots+Y_{t-i}\right)+1
$$

Applying the two relations we have defined between coordinates, and calling $\langle t\rangle$ the least residue of $t \bmod i$, we see that

$$
\begin{aligned}
-\left(Y_{1}+Y_{2}+\cdots+Y_{t-i}\right)+1 & =-\left(Y_{1}+Y_{2}+\cdots+Y_{t-2 i}+p_{t-i}\right)+1 \\
& =-\left(Y_{1}+Y_{2}+\cdots+Y_{t-3 i}+p_{t-2 i}+p_{t-i}\right)+1 \\
& =-\left(Y_{1}+Y_{2}+\cdots+Y_{\langle t\rangle}+\sum_{\substack{i<j \leqslant n-i \\
j \equiv t \bmod i}} p_{j}\right)+1 \\
& =-\left(\sum_{\substack{j \leqslant n-i \\
j \equiv t \bmod i}} p_{j}\right)+1 .
\end{aligned}
$$

Thus $p=Y_{1} w_{1}+Y_{2} w_{2}+\cdots+Y_{n-i} w_{n-i}+\alpha_{n-i+1, n}$ and so $w_{1}, \ldots, w_{n-i}$ form a lattice basis of $A$. Thus the vertices of $\mathcal{P}_{n}\left(\mathcal{I}_{i}\right)$ form a lattice basis, and so $\mathcal{P}_{n}\left(\mathcal{I}_{i}\right)$ is a unimodular simplex. 


\section{The first pyramidal interval vector polytope}

Recall that $\mathcal{P}_{n}\left(\mathcal{I}_{1, n-1}\right)$ is the convex hull of all vectors in $\mathbb{R}^{n}$ with interval length 1 or $n-1$. For example,

$$
\mathcal{P}_{4}\left(\mathcal{I}_{1,3}\right)=\operatorname{conv}((1,0,0,0),(0,1,0,0),(0,0,1,0),(0,0,0,1),(1,1,1,0),(0,1,1,1)),
$$

whose flow-dimension graph is depicted in Figure 2.

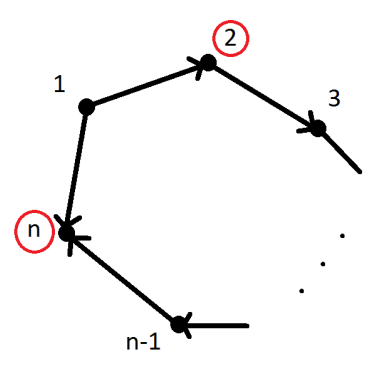

Figure 2: $G_{\mathcal{P}_{n}\left(\mathcal{I}_{1, n-1}\right)}$.

Proposition 9. The dimension of $\mathcal{P}_{n}\left(\mathcal{I}_{1, n-1}\right)$ is $n$.

Proof. The affine hull of $e_{1}, \ldots, e_{n}$ is the $(n-1)$-dimensional set

$$
\left\{x \in \mathbb{R}^{n} \mid x_{1}+\cdots+x_{n}=1\right\} .
$$

Since $\alpha_{1, n-1}$ is not in this set, $\operatorname{dim}\left(\mathcal{P}_{n}\left(\mathcal{I}_{1, n-1}\right)\right)=n$.

Recall that the $f$-vector of a polytope tells us the number of faces the polytope has of each dimension. Our next task is to compute the $f$-vector of $\mathcal{P}_{n}\left(\mathcal{I}_{1, n-1}\right)$.

Lemma 10. Let $n \geqslant 3$. Then $\mathcal{B}:=\operatorname{conv}\left(e_{1}, e_{n}, \alpha_{1, n-1}, \alpha_{2, n}\right)$ is a 2-dimensional face of $\mathcal{P}_{n}\left(\mathcal{I}_{1, n-1}\right)$.

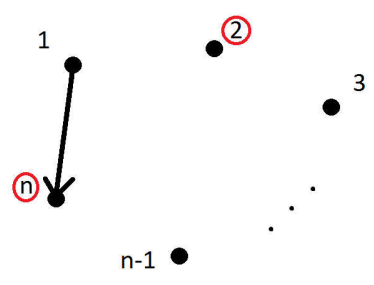

Figure 3: $G_{\mathcal{A}}$. 
Proof. We first consider $\mathcal{A}=\operatorname{conv}\left(e_{n}, \alpha_{1, n-1}, \alpha_{2, n}\right)$. The corresponding elementary vectors of the vertex set are $\left\{e_{1, n}, e_{2}, e_{n}\right\}$. So we build the flow-dimension graph as seen in Figure $2, G_{\mathcal{A}}=(V, E)$ where $V=[n], E=\{(1, n)\}$ corresponding to $e_{1, n}$. The subset $V_{1}=\{2, n\}$ (circled in Figure 2) corresponds to $e_{2}$ and $e_{n}$. This graph has $n-1$ connected components, two of which contain elements of $V_{1}$ so that $k_{0}=n-3$.

If we let $\lambda_{1} e_{n}+\lambda_{2} \alpha_{1, n-1}+\lambda_{3} \alpha_{2, n}=\mathbf{0}$, we first notice that $\lambda_{2}=0$ since $\alpha_{1, n-1}$ is the only vector with a nonzero first coordinate. But this implies that $\lambda_{1}=\lambda_{3}=0$. Since the coefficients cannot sum to 1 , we conclude that $\mathbf{0} \notin \operatorname{aff}\left(e_{n}, \alpha_{1, n-1}, \alpha_{2, n}\right)$. So now by Theorem 6,

$$
\operatorname{dim}\left(\operatorname{conv}\left(e_{n}, \alpha_{1, n-1}, \alpha_{2, n}\right)\right)=n-k_{0}-1=n-(n-3)-1=2 .
$$

Finally $e_{1}=(1) \alpha_{1, n-1}+(-1) \alpha_{2, n}+(1) e_{n}$ is in the affine hull of $\mathcal{A}$ and thus does not add a dimension. We conclude that $\operatorname{dim}(\mathcal{B})=2$.

Corollary 11. Let $\mathcal{I}:=\left\{e_{1}, e_{2}, \ldots, e_{n}, \alpha_{1, n-1}, \alpha_{2, n}\right\}$. For $2 \leqslant i \leqslant n-1$ each $e_{i}$ adds a dimension to $\mathcal{P}_{n}\left(\mathcal{I}_{1, n-1}\right)$, that is, $e_{i} \notin \operatorname{aff}\left(\mathcal{I} \backslash\left\{e_{i}\right\}\right)$.

Proof. This follows from Proposition 9 and Lemma 10. Since $\mathcal{B}$ has dimension 2 and $\mathcal{P}_{n}\left(\mathcal{I}_{1, n-1}\right)$ has dimension $n$, then the $n-2$ remaining vertices must add the remaining $n-2$ dimensions.

Lemma 12. Let $\mathcal{B}$ as in Lemma 10. Then $\mathcal{B}$ has $f$-vector $(1,4,4,1)$.

Proof. Since $\mathcal{B}$ has dimension $2, f_{1}=f_{0}$. We know that $\left\{e_{n}, \alpha_{1, n-1}, \alpha_{2, n}\right\}$ are three vertices of $\mathcal{B}$. If $e_{1} \in \operatorname{conv}\left(e_{n}, \alpha_{1, n-1}, \alpha_{2, n}\right)$ then

$$
e_{1}=\lambda_{1} e_{n}+\lambda_{2} \alpha_{1, n-1}+\lambda_{3} \alpha_{2, n}
$$

where the coefficients sum to 1 . Since $\alpha_{1, n-1}$ is the only vector with a nonzero coordinate in the first position, $\lambda_{2}=1$. This in turn implies that $\lambda_{1}=\lambda_{3}=0$, contradicting (2). So $e_{1} \notin \operatorname{conv}\left(e_{n}, \alpha_{1, n-1}, \alpha_{2, n}\right)$ and therefore forms a fourth vertex.

We can tie all this together with the following theorem. First we define a d-pyramid $P$ as the convex hull of a $(d-1)$-dimensional polytope $K$ (the basis of $P$ ) and a point $A \notin \operatorname{aff}(K))$ (the apex of $P$ ).

Theorem 13 (see, e.g., [7]). If $P$ is a d-pyramid with basis $K$ then

$$
\begin{aligned}
f_{0}(P) & =f_{0}(K)+1 \\
f_{k}(P) & =f_{k}(K)+f_{k-1}(K) \quad \text { for } 1 \leqslant k \leqslant d-2 \\
f_{d-1}(P) & =1+f_{d-2}(K) .
\end{aligned}
$$

We notice that the rows of Pascal's 3-triangle act in the same manner and we claim the face numbers for $\mathcal{P}_{n}\left(\mathcal{I}_{1, n-1}\right)$ can be derived from Pascal's 3-triangle. 
Proof of Theorem 4. Recall that $\mathcal{I}=\left\{e_{1}, e_{2}, \ldots, e_{n}, \alpha_{1, n-1}, \alpha_{2, n}\right\}$ is the vertex set for $\mathcal{P}_{n}\left(\mathcal{I}_{1, n-1}\right)$ with $n \geqslant 3$, and let $\mathcal{R}_{k}:=\operatorname{conv}\left(\mathcal{I} \backslash\left\{e_{k}, e_{k+1}, \ldots, e_{n-1}\right\}\right)$ for $1 \leqslant k<n$. Then it is clear that $\mathcal{P}_{n}\left(\mathcal{I}_{1, n-1}\right)$ is the convex hull of the union of the $(n-1)$-dimensional polytope $\mathcal{R}_{n-1}$ and $e_{n-1} \notin \operatorname{aff}\left(\mathcal{R}_{n-1}\right)$ (by Corollary 11), and thus is a pyramid and its face numbers can be computed as in Theorem 13 from the face numbers of $\mathcal{R}_{n-1}$.

Notice next that $\mathcal{R}_{n-1}$ is the convex hull of the $(n-2)$-dimensional polytope $\mathcal{R}_{n-2}$ and $e_{n-2} \notin$ aff $\left(\mathcal{R}_{n-2}\right)$ (again by Corollary 11 ), so we can compute the face numbers of $\mathcal{R}_{n-1}$ from those of $\mathcal{R}_{n-2}$ as in Theorem 13 .

We can continue this process until we get that $\mathcal{R}_{3}$ is the convex hull of $\mathcal{R}_{2}$ and $e_{2} \notin \operatorname{aff}\left(\mathcal{R}_{2}\right)$. But we notice that $\mathcal{R}_{2}=\mathcal{B}$, so by Lemma $12, f_{0}\left(\mathcal{R}_{2}\right)=f_{1}\left(\mathcal{R}_{2}\right)=4$. From here we can build the $f$-vector of $\mathcal{P}_{n}\left(\mathcal{I}_{1, n-1}\right)$ recursively, using Theorem 13.

Our next goal is to compute the volume of $\mathcal{P}_{n}\left(\mathcal{I}_{1, n-1}\right)$. A simple induction proof gives:

Lemma 14. The determinant of the $n \times n$-matrix

$$
\left[\begin{array}{ccccc}
0 & 1 & 1 & \cdots & 1 \\
1 & 0 & 1 & \cdots & 1 \\
& & \ddots & & \\
1 & \cdots & 1 & 0 & 1 \\
1 & 1 & \cdots & 1 & 0
\end{array}\right]
$$

is $(-1)^{n-1}(n-1)$.

Proof of Theorem 5. In order to calculate the volume of $\mathcal{P}_{n}\left(\mathcal{I}_{1, n-1}\right)$ we will first triangulate the 2-dimensional base of the pyramid $\mathcal{B}$ from Lemma 10: namely, $\mathcal{B}$ is the union of

$$
\triangle_{1}=\operatorname{conv}\left(e_{1}, e_{n}, \alpha_{1, n-1}\right) \quad \text { and } \quad \triangle_{2}=\operatorname{conv}\left(e_{n}, \alpha_{1, n-1}, \alpha_{2, n}\right) .
$$

By Corollary 11, each $e_{2}, \ldots, e_{n-1}$ adds a dimension so that the convex hull of these points and $\triangle_{1}$ is an $n$-dimensional simplex. The same can be said of $\triangle_{2}$. Call these simplices $S_{1}$ and $S_{2}$ respectively; thus $S_{1}$ and $S_{2}$ triangulate $\mathcal{P}_{n}\left(\mathcal{I}_{1, n-1}\right)$, and the sum of their volumes is equal to the volume of $\mathcal{P}_{n}\left(\mathcal{I}_{1, n-1}\right)$. In order to calculate the volume of $S_{1}$ and $S_{2}$, we will use the Cayley Menger determinant [3]. Consider $S_{1}$, whose volume is the determinant of the matrix

$$
\left[\begin{array}{llll}
e_{1}-\alpha_{1, n-1} & e_{2}-\alpha_{1, n-1} & \cdots & e_{n}-\alpha_{1, n-1}
\end{array}\right]=\left[\begin{array}{cccccc}
0 & -1 & -1 & \cdots & -1 & -1 \\
-1 & 0 & -1 & \cdots & -1 & -1 \\
-1 & -1 & 0 & -1 & \cdots & -1 \\
& & & \ddots & & \\
-1 & -1 & \cdots & -1 & 0 & -1 \\
0 & 0 & 0 & \cdots & 0 & 1
\end{array}\right] \text {. }
$$


Cofactor expansion on the last row will leave us with the determinant, up to a sign, of the $(n-1) \times(n-1)$ matrix

$$
\left[\begin{array}{ccccc}
0 & -1 & -1 & \cdots & -1 \\
-1 & 0 & -1 & \cdots & -1 \\
& & \ddots & & \\
-1 & \cdots & -1 & 0 & -1 \\
-1 & -1 & \cdots & -1 & 0
\end{array}\right]
$$

which, when ignoring sign, by Lemma 14 is $n-2$. Therefore the volume of $S_{1}$ is $n-2$.

A similar computation gives the volume of $S_{2}$ as $n-2$, and so the volume of $\mathcal{P}_{n}\left(\mathcal{I}_{1, n-1}\right)$ is $2(n-2)$, as desired.

\section{The $i^{\text {th }}$ pyramidal interval vector polytope}

Recall that the $i^{\text {th }}$ pyramidal interval vector polytope is $\mathcal{P}_{n}\left(\mathcal{I}_{1, n-i}\right)$, the convex hull of all interval vectors in $\mathbb{R}^{n}$ with interval length 1 or $n-i$.

Example 15. For $n=6$ and $i=2$,

$$
\begin{aligned}
\mathcal{P}_{6}\left(\mathcal{I}_{1,4}\right)=\operatorname{conv} & ((1,0,0,0,0,0),(0,1,0,0,0,0),(0,0,1,0,0,0),(0,0,0,1,0,0) \\
& (0,0,0,0,1,0),(0,0,0,0,0,1),(1,1,1,1,0,0),(0,1,1,1,1,0) \\
& (0,0,1,1,1,1)) .
\end{aligned}
$$

The following proposition collects certain properties of $\mathcal{P}_{n}\left(\mathcal{I}_{1, n-i}\right)$. We omit its proof, since it is similar to the proofs in Section 5.

Proposition 16. The dimension of $\mathcal{P}_{n}\left(\mathcal{I}_{1, n-i}\right)$ is n. Furthermore, $\mathcal{P}_{n}\left(\mathcal{I}_{1, n-i}\right)$ can be constructed by taking iterative pyramids (with the sequence of top vertices $e_{i+1}, e_{i+2}, \ldots, e_{n-i}$ ) over the $2 i$-dimensional base

$$
\operatorname{conv}\left(\left\{e_{1}, e_{2}, \ldots, e_{i}, e_{n-i+1}, \ldots, e_{n}, \alpha_{1, n-i}, \alpha_{2, n-i-1} \ldots, \alpha_{i+1, n}\right\}\right)
$$

Using polymake to generate $f$-vectors for varying $n$, we observed that the $f$-vectors of $\mathcal{P}_{n}\left(\mathcal{I}_{1, n-i}\right)$ correspond to the sum of multiple shifted Pascal triangles; this is again due to its pyramid property. We also offer the following:

Conjecture 17. The volume of $\mathcal{P}_{n}\left(\mathcal{I}_{1, n-i}\right)$ equals $2^{i}(n-(i+1))$.

We conjecture something more concrete: namely, that $\mathcal{P}_{n}\left(\mathcal{I}_{1, n-i}\right)$ can be triangulated into $2^{i}$ simplices, and pyramiding over each of these simplices each yields a volume of $n-(i+1)$. 


\section{Acknowledgements}

We thank the creators and maintainers of the software packages polymake [6] and LattE $[4,8]$, which were indispensable for our project. We also thank an anonymous referee for helpful suggestions, and Ricardo Cortez and the staff at MSRI for creating an ideal research environment at MSRI-UP. This research was partially supported by the NSF through the grants DMS-1162638 (Beck) and DMS-1156499 (MSRI-UP REU), and by the NSA through grant H98230-11-1-0213.

\section{References}

[1] Matthias Beck and Sinai Robins, Computing the continuous discretely: Integer-point enumeration in polyhedra, Undergraduate Texts in Mathematics, Springer, New York, 2007, Electronically available at http://math.sfsu.edu/beck/ccd.html.

[2] Geir Dahl, Polytopes related to interval vectors and incidence matrices, Linear Algebra Appl. 435 (2011), no. 11, 2955-2960. Correction in: Linear Algebra Appl. 438 (2013), no. 1, 1-6.

[3] Carlos D'Andrea and Martín Sombra, The Cayley-Menger determinant is irreducible for $n \geqslant 3$, Sibirsk. Mat. Zh. 46 (2005), no. 1, 90-97.

[4] Jesús A. De Loera, Raymond Hemmecke, Jeremiah Tauzer, and Ruriko Yoshida, Effective lattice point counting in rational convex polytopes, J. Symbolic Comput. 38 (2004), no. 4, 1273-1302.

[5] Eugène Ehrhart, Sur les polyèdres rationnels homothétiques à $n$ dimensions, C. R. Acad. Sci. Paris 254 (1962), 616-618.

[6] Ewgenij Gawrilow and Michael Joswig, polymake: a framework for analyzing convex polytopes, Polytopes - combinatorics and computation (Oberwolfach, 1997), DMV Sem., vol. 29, Birkhäuser, Basel, 2000, pp. 43-73. Software polymake available at http: //www . polymake .org.

[7] Branko Grübaum, Convex polytopes, second ed., Graduate Texts in Mathematics, vol. 221, Springer-Verlag, New York, 2003, Prepared and with a preface by Volker Kaibel, Victor Klee and Günter M. Ziegler.

[8] Matthias Köppe, A primal Barvinok algorithm based on irrational decompositions, SIAM J. Discrete Math. 21 (2007), no. 1, 220-236 (electronic), arXiv:math.C0/0603308. Software LattE macchiato available at http://www . math. ucdavis.edu/〜mkoeppe/latte/.

[9] Alexander Postnikov, Permutohedra, associahedra, and beyond, Int. Math. Res. Not. (2009), no. 6, 1026-1106.

[10] Neil J. A. Sloane, On-line encyclopedia of integer sequences, http://oeis.org/. 